\title{
Patterned Ultra-Thin Gold Nanostructures on Graphene
}

\author{
Georg Zagler $^{1 *}$, Clemens Mangler ${ }^{1}$, Jani Kotakoski ${ }^{1}$ \\ 1. University of Vienna, Faculty of Physics, \\ Boltzmanngasse 5, 1090, Vienna, Austria \\ * Corresponding author: georg.zagler@univie.ac.at
}

Focused electron-beam deposition is a convenient addition to state-of-the-art resist-based patterning techniques. It employs local chemical vapor deposition (CVD) from a precursor gas induced by the beam, allowing the growth of nm scale features [1]. In a transmission electron microscope this can be used to create patterns on the nm scale with the readily available hydrocarbon contamination [2]. Here we present a novel technique to grow gold nanoparticles (NPs) on $\mu \mathrm{m}$-scale clean graphene. Combining top-down electron-beam induced deposition of hydrocarbon contamination onto two-dimensional materials, with the bottom-up growth of gold NPs, we obtain patterns of gold NPs while introducing no additional contamination.

Commercially available graphene, grown by CVD and covered with a sacrificial layer, is put on a silicon-nitride TEM-grid. The sacrificial layer is then removed by thermal treatment. For all subsequent steps the specimen is loaded in our ultra-high vacuum (UHV) system connected to a Nion UltraSTEM 100 in Vienna. To remove hydrocarbon contamination that exists practically on all graphene samples, the specimen is cleaned in the microscope column with a laser [3]. That leads to large areas $(\sim \mu \mathrm{m} 2)$ of pristine graphene. After this, electron-beam deposition with the 1 - $\AA$-sized probe is used to make patterns of pinned-down hydrocarbon contamination on graphene. For the deposition of gold, the sample is transferred in UHV to a thermal evaporator. The gold (Goodfellow $99.999 \%$ purity) is evaporated at $1080{ }^{\circ} \mathrm{C}$ for 30 minutes at a distance of circa $15 \mathrm{~cm}$ to the specimen. After the deposition the specimen is transported to the microscope for imaging.

In figure 1a the specimen is depicted after laser-cleaning and pattern creation. Small bright clusters can be seen prior to gold evaporation. They are most likely silicon from the synthesis or transfer processes, that are excited by the laser treatment and thereby form clusters, either at holes or at grain boundaries or overlapping graphene sheets. The other bright contrast stems from the pinned-down contamination. In figure $1 \mathrm{~b}$ the result of the evaporation can be seen. Gold was only found along overlapping graphene, at previously existing highly active sites such as holes or grain boundaries, and on pinned-down contamination. This is consistent with previous reports [4]. Here we found the also non-pinned-down contamination does not foster gold nucleation (see the weak contrast in figure 1b). This allows for a precise patterning with gold NPs on pristine graphene.

We developed this method for in-situ atomic resolution studies, where no additional components are introduced, yielding low levels of contamination. Provided an electron source, a laser and a thermal evaporator are connected within a UHV system, it effortlessly allows for nm-scale precision of patterning and growth with just a few steps. This method could also be used to create transferable patterns, creating contacts by partially growing gold on pristine graphene or to create patterns, e.g. for plasmonics. This technique could also be applied to other materials such as silver. 
References:

[1] I. Utke et al. J. Vac. Sci. Technol. B, 18 (2000), p:182-189.

[2] J. C. Meyer et al. Appl. Phys. Lett., 92 (2008), p:3-6.

[3] M. Tripathi et al. Phys. Status Solidi RRL 11 (2017), 1700124.

[4] R. Zan et al. Small, 7 (2011), p:2868-2872.

[5] Georg Zagler acknowledges the Vienna Doctoral School in Physics for their support.
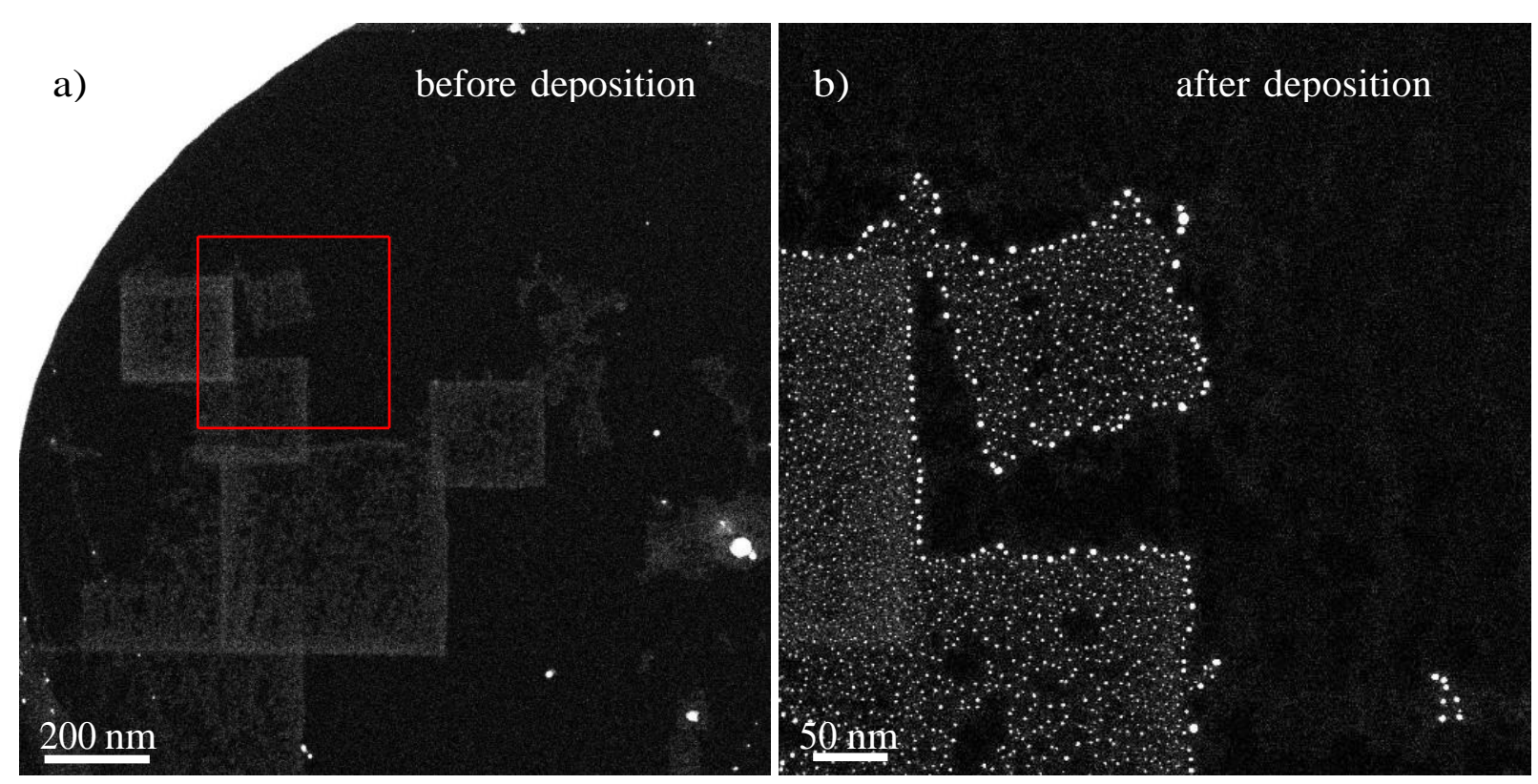

Figure 1. Patterns of gold nanoparticles on a suspended graphene sheet. Panel a) displays patches of hydrocarbon contamination pinned down by the electron beam. Panel b) displays a part of the area of panel a) (marked in red) after the thermal deposition of gold, creating patches of gold nanoparticles. 